\title{
'Malnutrition Universal Screening Tool' predicts mortality and length of hospital stay in acutely ill elderly
}

\author{
Rebecca J. Stratton*, Claire L. King, Mike A. Stroud, Alan A. Jackson and Marinos Elia \\ Institute of Human Nutrition, School of Medicine, University of Southampton, Level F (MP 113), Southampton General Hospital, \\ Southampton SO16 6YD, UK
}

(Received 27 April 2005 - Revised 2 September 2005 - Accepted 16 September 2005)

\begin{abstract}
Malnutrition and its impact on clinical outcome may be underestimated in hospitalised elderly as many screening procedures require measurements of weight and height that cannot often be undertaken in sick elderly patients. The 'Malnutrition Universal Screening Tool' ('MUST') has been developed to screen all adults, even if weight and/or height cannot be measured, enabling more complete information on malnutrition prevalence and its impact on clinical outcome to be obtained. In the present study, 150 consecutively admitted elderly patients (age 85 (SD 5.5) years) were recruited prospectively, screened with 'MUST' and clinical outcome recorded. Although only $56 \%$ of patients could be weighed, all ( $n$ 150) could be screened with 'MUST'; $58 \%$ were at malnutrition risk and these individuals had greater mortality (in-hospital and post-discharge, $P<0 \cdot 01)$ and longer hospital stays $(P=0.02)$ than those at low risk. Both 'MUST' categorisation and component scores (BMI, weight loss, acute disease) were significantly related to mortality $(P<0.03)$. Those patients with no measured or recalled weight ('MUST' subjective criteria used) had a greater risk of malnutrition $(P=0 \cdot 01)$ and a poorer clinical outcome $(P<0.002)$ than those who could be weighed and, within both groups, clinical outcome was worse in those at risk of malnutrition. The present study suggests that 'MUST' predicts clinical outcome in hospitalised elderly, in whom malnutrition is common (58\%). In those who cannot be weighed, a higher prevalence of malnutrition and associated poorer clinical outcome supports the importance of routine screening with a tool, like 'MUST', that can be used to screen all patients.
\end{abstract}

Malnutrition: Screening: Elderly: Validity: Outcome assessment

Malnutrition is common, under-recognised and under-treated across specialities, including elderly care (Mowe \& Bohmer, 1991, McWhirter \& Pennington, 1994, Morley, 1998, Kelly et al. 2000, Waitzberg et al. 2001, Stratton et al. 2003b). Several studies have surprised health workers by revealing that about two-thirds of patients with malnutrition, including elderly patients, were unrecognised (Mowe \& Bohmer, 1991; Kelly et al. 2000). To combat this problem many national and international organisations have recommended that nutritional screening should be routinely undertaken in hospitalised patients to identify those likely to benefit from nutritional intervention: British Dietetic Association (1999), Department of Health (2001), Council of Europe (2002), British Association for Parenteral and Enteral Nutrition (Elia, 2000, 2003), ESPEN (Kondrup et al. 2003), ASPEN (1995), Royal College of Physicians (2002) and NHS Quality Improvement Scotland (2003). Screening has become a mandatory standard in some countries (NHS Quality Improvement Scotland, 2003) and so the choice of a screening tool is obviously an important issue. There are many valuable tools that have been developed, validated and are widely used for the detection of malnutrition in clinical practice, including Subjective Global Assessment (Detsky et al. 1987), MiniNutritional Assessment (MNA; Guigoz et al. 1996) and its short form (SF-MNA; Murphy et al. 2000), Nutrition Risk
Screening (Kondrup et al. 2003) and 'Malnutrition Universal Screening Tool' ('MUST'; Elia, 2003). Amongst the desirable characteristics of screening tools are that they are easy and quick to perform, particularly in busy hospital wards, acceptable to patients and health care workers, and have good reproducibility and validity. An important type of validity is predictive validity or the ability to predict clinical outcome, with its economic implications. Although many studies have linked malnutrition, identified by a variety of screening tools, to poor clinical outcome (for a review, see Elia et al. 2005), the available literature on the predictive validity of nutrition screening tools for elderly hospitalised patients is inadequate for at least two reasons. First, there is a notable lack of information about the value of simple quick screening tools for predicting clinically relevant outcome measures. Although both the full MNA (Guigoz et al. 1996) and the Subjective Global Assessment (Detsky et al. 1987) have been related to outcome, they can be lengthy to undertake and potentially impractical for routine use by a variety of health care workers in busy clinical wards (Stratton et al. 2004), which is partly why a shorter form of the MNA was developed (Murphy et al. 2000). Second, a number of tools (e.g. MNA (Guigoz et al. 1996), SF-MNA (Murphy et al. 2000), Nutrition Risk Screening (Kondrup et al. 2003), Nutrition Risk Score (Reilly et al. 1995)) do not explicitly state how to use the 
tool in those patients who cannot have measurements of weight and/or height undertaken. Similarly, these tools do not appear to have been validated using any such alternatives (e.g. use of recalled values or subjective criteria). For some hospitalised patients, particularly elderly care patients, it may be difficult or impossible to obtain measurements of weight or height. Studies in acute hospitals have failed to establish malnutrition risk in up to $56 \%$ of patients because of difficulties in measuring weight and/or height (particularly in medical patients) (Kelly et al. 2000; Powell-Tuck \& Hennessy, 2003). A particularly high figure may be expected in elderly care wards that manage severely frail, disabled individuals who are often bed-bound. Consequently, many studies lack complete information on the prevalence of malnutrition and the associated clinical outcome of this potentially vulnerable patient group. In contrast to many screening tools that require measurement of weight to be undertaken to establish malnutrition risk, 'MUST' (Elia, 2003; www.bapen.org.uk) for adults has been developed as a tool that can establish malnutrition risk in all adult patients, even in those in whom weight and/or height cannot be measured. It does this by using recalled or surrogate measurements for weight, height or BMI, and other more subjective criteria when necessary. Previous work has established that correct categorisation of patients whose weight (and/or height) could not be measured could be established with a sensitivity and specificity of $\geq 95 \%$ (Elia, 2003). Studies have also indicated the concurrent validity of 'MUST' with other screening tools (including MNA, SF-MNA and Subjective Global Assessment) in use in clinical practice (Stratton et al. 2004). This investigation had three aims: (1) to test the hypothesis that 'MUST' could be undertaken on all admissions to elderly care wards, enabling malnutrition prevalence to be established for everyone, including those who could not be weighed (and hence screened by other commonly used tools); (2) to assess the predictive validity of 'MUST' with regard to clinical outcome in the group as a whole and in the subgroup of patients who could not be weighed; (3) to investigate whether malnutrition and poor clinical outcome were more prevalent in elderly patients in whom weight (measured or recalled) could not be obtained.

\section{Subjects and methods}

Local Research Ethics Committee approval was obtained. Patients gave consent before being screened and $<2 \%$ of patients declined to participate. Included in the study were 150 consecutive emergency admissions (100 female, fifty male) from home and care homes to two elderly care wards (mean age 85 (SD 5.5) years; see Table 1). Reasons for admission included falls/collapse $(25 \%)$, cardiac disease $(16 \%)$, cerebrovascular accident $(14 \%)$, respiratory disease $(11 \%)$ and a range of other conditions (gastrointestinal complaints, renal failure, prostate cancer, spinal disorders).

\section{'MUST' methodology}

'MUST' (Fig. 1, see www.bapen.org.uk for free download of tool and explanatory booklet; Todorovic et al. 2003) was completed by an experienced research dietitian (C. K.) on patients within $48-72 \mathrm{~h}$ of admission to hospital as follows.
Table 1. Patient characteristics according to malnutrition risk

\begin{tabular}{lcccc}
\hline & All & Low & Medium & High \\
\hline$n$ & 150 & 63 & 25 & 62 \\
$(\%)$ & 100 & 42 & 17 & 41 \\
Mean age in years & 85 & 85 & 86 & 86 \\
Mean age SD & 5.5 & 4.6 & 4.5 & 6.6 \\
No. of males & 50 & 16 & 10 & 24 \\
No. of females & 100 & 47 & 15 & 38 \\
Percentage admitted from & 17 & 9.5 & 8 & 29 \\
$\quad$ nursing/residential care* & & & & \\
\hline
\end{tabular}

${ }^{*}$ Three malnutrition categories (low, medium, high), $P=0.006 ; \chi^{2} 10 \cdot 1$.

Height was measured to the nearest $0 \cdot 1 \mathrm{~cm}$ using a portable, free-standing stadiometer (Seca, Leicester, UK), according to standard methodology (Elia, 2003). If height could not be measured accurately (e.g. patient unable to stand), recalled height (if reliable and realistic) (Elia, 2003; Stratton et al. $2003 a$ ) or surrogate measures (e.g. knee height) (Elia, 2000, 2003) were used to calculate height. Weight was measured to the nearest $0.01 \mathrm{~kg}$ using Seca clinical scales (conforming to EU Directive 90/384/EEC). If weight could not be measured accurately, recalled weight (if reliable and realistic) was used (Elia, 2003; Stratton et al. 2003a). BMI $\left(\mathrm{kg} / \mathrm{m}^{2}\right)$ was calculated and scored accordingly. If neither weight nor height could be obtained, subjective criteria assessing physical appearance (very thin, thin etc.), were used. Percentage unplanned weight loss over 3-6 months was calculated, either from documented weights in patients' notes or from patients' reports, and scored accordingly. Subjective criteria could be used if reliable records or reports could not be obtained (Elia, 2003). It was noted if there had been or was likely to be no nutritional intake for $>5 \mathrm{~d}$ (termed an 'acute disease effect' in the tool) and this was scored. For a detailed explanation of the methods and evidence base for 'MUST' and interpretation of 'acute disease effect', see Elia (2003), Todorovic et al. (2003) and www.bapen.org.uk.

A record was made of the proportion of patients who could be screened with 'MUST' and the prevalence of malnutrition risk (low, medium, high; or combined into two risk categories (low and medium + high risk)). The patients who could not be weighed or have their height measured were identified and their 'MUST' malnutrition risk established using recalled/ documented values, surrogate measures and subjective criteria. The time taken to complete screening was recorded and the ease of use on a four-point Likert scale (very easy, easy, difficult, very difficult) assessed. At the time of the study, nutritional screening was not routinely carried out on these wards. The results of screening in the present study were not divulged to the nursing staff so that care continued routinely according to local policy.

\section{Predictive validity}

To assess predictive validity, length of hospital stay (days), inhospital mortality, discharge destination (e.g. own home, residential home, nursing home), post-discharge mortality (3- and 6-month) and hospital readmission rates (3 month) were prospectively assessed for each patient and related to 'MUST' categorisation. 


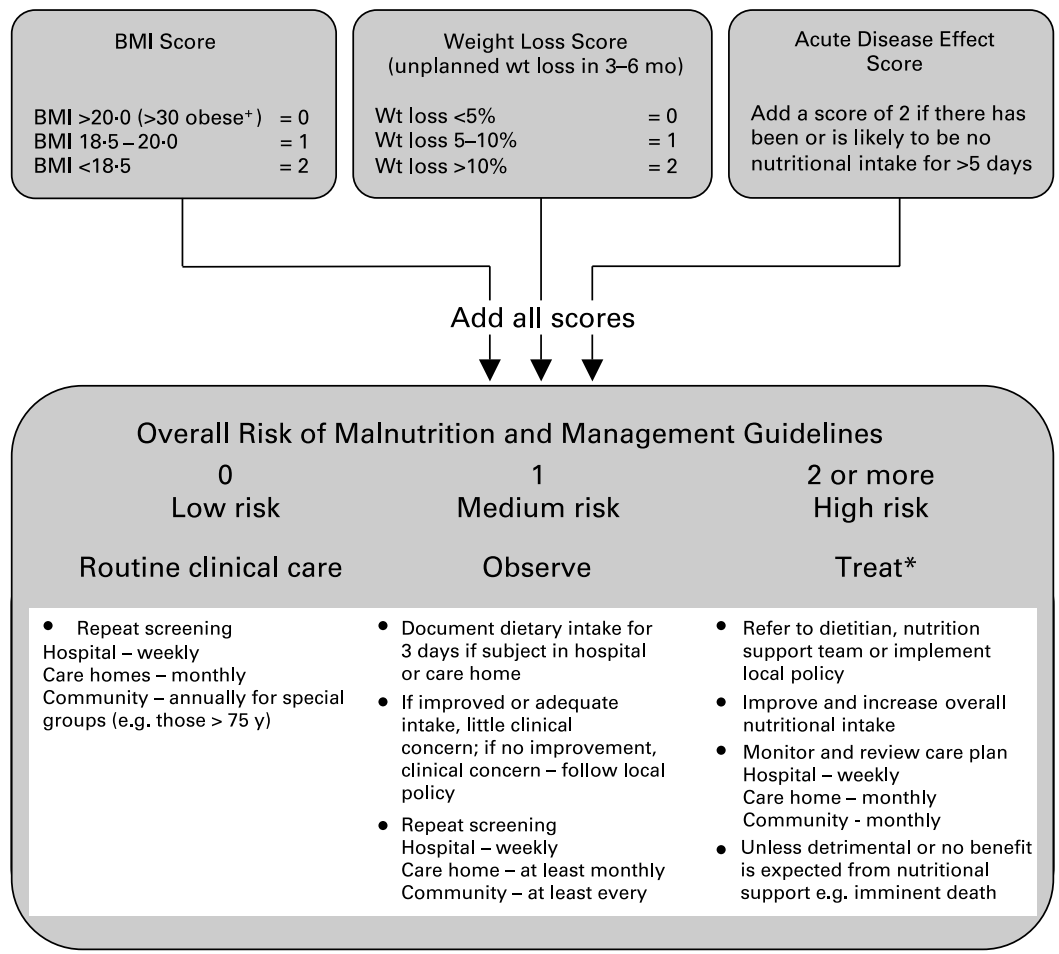

Record malnutrition risk category, presence of obesity and/or need for special diets and follow local policy. Reassess those identified at risk as they move through care settings. +In the obese, underlying acute conditions are generally controlled before the treatment of obesity.

If unable to obtain height and weight, alternative measurements and subjective criteria are provided (Elia, 2003).

(C) BAPEN 2003

Fig. 1. 'Malnutrition Universal Screening Tool'.

\section{Statistical analysis}

ANOVA was used to compare mean results or the KruskalWallis one-way ANOVA test was undertaken if data were not normally distributed. Length of hospital stay and readmissions were assessed using Kaplan-Meier Survival Analysis and the log rank test to take into account mortality (similar results (not reported) were obtained by excluding patients who died from analysis using Kruskal-Wallis ANOVA). Differences in proportions were analysed using $\chi^{2}$ and the influence of other variables (e.g. age) was assessed using binary logistic regression. Data are presented as means and standard deviations or median and range unless otherwise indicated. Analysis was undertaken using SPSS statistical software package version 11.0 (SPSS, Woking, Surrey, UK).

\section{Results}

Practicality of 'MUST' and prevalence of malnutrition risk

All patients ( $n$ 150) were screened using 'MUST' enabling malnutrition risk and the prevalence of malnutrition to be calculated for the whole group. Most patients $(58 \%, n$ 87) were at risk of malnutrition (17\% ( $n$ 25) medium and $41 \%(n 62)$ high risk using 'MUST'); $28 \%$ ( $n$ 42) of patients had a $\mathrm{BMI}<20 \mathrm{~kg} / \mathrm{m}^{2}$ (BMI score $\left.\geq 1\right) ; 35 \%$ (n 52) had $>5 \%$ unintentional weight loss in 3-6 months (weight loss score $\geq 1$ ); $25 \%$ had an 'acute disease effect' (score 2). There were no significant differences between low-, medium- and high-risk patients with regard to age or the proportion of men and women but significantly more patients who were at malnutrition risk $(23 \%$ of medium + high-risk group v. $9 \%$ of low-risk group) were admitted from institutions (nursing and residential homes) than from home (Table 1). During admission, there was little overall change in weight (for patients who could be weighed on both admission and discharge, +0.41 (SD 2.84) kg, $n$ 79) and no significant difference between patients who were $(+0.58(\mathrm{SD} 3.5) \mathrm{kg})$ or were not $(+0.18(\mathrm{SD} 3.5) \mathrm{kg})$ at risk of malnutrition on admission.

During screening with 'MUST' (within $72 \mathrm{~h}$ of admission), weight could be measured in only $56 \%(n 84)$ of patients. In a further $21 \%$ ( $n$ 31) weight was obtained from reliable recall. In these 115 patients, measurement of height could only be undertaken in $16 \%(n 18)$ and in most cases was obtained from reliable recall or recent documentation $(n 58)$ and surrogate measures ( $n$ 39) (measurements of height not undertaken if data on weight could not be obtained). For thirty-five patients ( $23 \%$ of group; main diagnoses cerebrovascular accident, falls, extreme frailty with severe chest infection), no data on weight could be obtained from measurements or recalled values and so 'MUST' subjective criteria were used to categorise malnutrition risk. 'MUST' was rated as 'easy' to use and took approximately $3-5 \mathrm{~min}$ per patient.

\section{Predictive validity of 'MUST'}

Mortality and length of stay. In-hospital mortality rose significantly with increasing malnutrition risk category (Fig. 2). 

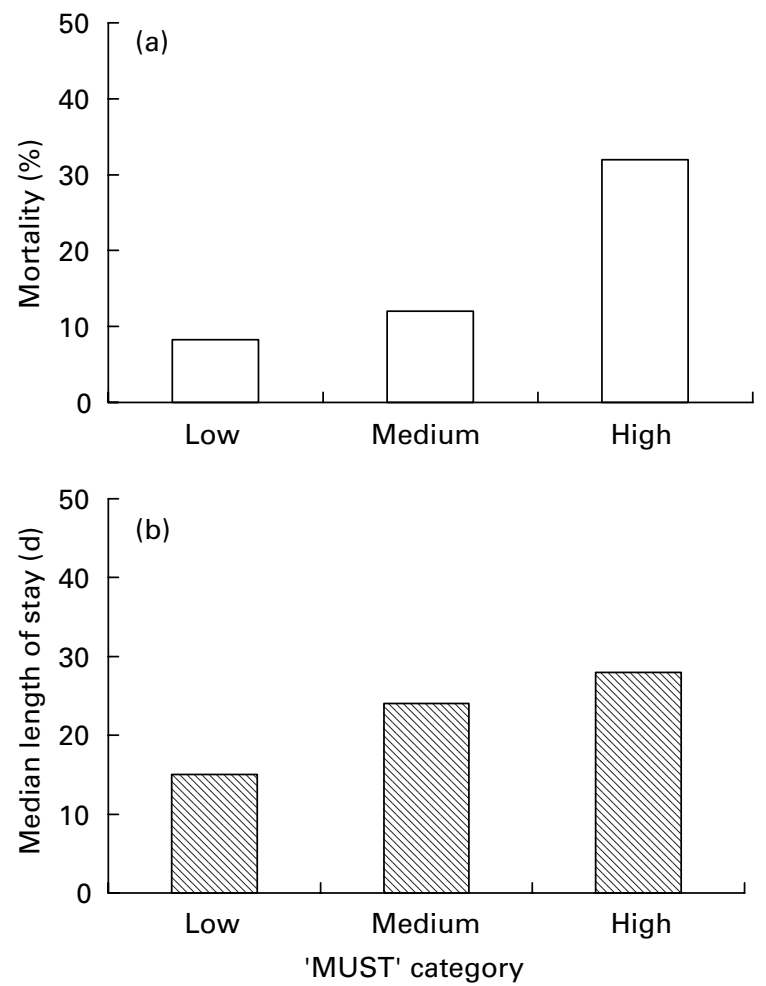

Fig. 2. Clinical outcome according to malnutrition risk with the 'Malnutrition Universal Screening Tool' ('MUST'). (a), In-hospital mortality $(P=0.01)$; (b), length of hospital stay $(P=0.02)$. For details of procedures, see p. 326.

For those alive on leaving hospital ( $n$ 119), mortality 3 months (12\% (14 deaths)) and 6 months (21\% (25 deaths)) after discharge was also significantly greater for medium- and highrisk patients $(5 \%$ and $24 \%$ at 3 months $(P=0.01) ; 9 \%$ and $28 \%$ at 6 months $(P=0.002)$, respectively) compared with low-risk patients (5\% at 3 months and $13 \%$ at 6 months). Length of hospital stay increased progressively with malnutrition risk category (low-risk median $15 \mathrm{~d}$ (95\% CI 11, $19 \mathrm{~d})$; medium risk $24 \mathrm{~d}$ (95\% CI 16, $32 \mathrm{~d}$ ); high risk $28 \mathrm{~d}$ (95\% CI 21, 35d); $P=0 \cdot 02$, Kaplan-Meier and log rank test) (see Fig. 2). (The length of stay of patients who died did not differ significantly according to 'MUST' category; Kruskal-Wallis ANOVA.)

The individual components of 'MUST' also predicted clinical outcome (Fig. 3). Individuals with a BMI $<20 \mathrm{~kg} / \mathrm{m}^{2}$ (BMI score 1 or more) had a significantly greater in-hospital mortality than those with a BMI $>20 \mathrm{~kg} / \mathrm{m}^{2}$ (BMI score 0) ( $31 \%$ v. $\left.14.3 \%, P=0.02, \chi^{2} 5 \cdot 40\right)$. Similarly, mortality was significantly greater in individuals with $>5 \%$ unintentional weight loss over 3-6 months (weight loss score 1 or more) than in those with $<5 \%$ loss (weight loss score 0$)(30.8 \%$ v. $\left.12.6 \%, P=0.007, \chi^{2} 7 \cdot 17\right)$. Patients with an 'acute disease effect' also had significantly greater mortality than those without (36.8\% v. $\left.12.8 \%, P=0.001, \chi^{2} 10 \cdot 52\right)$. Using binary logistic regression, each of the individual component scores (BMI, weight loss, 'acute disease effect') had a significant effect on mortality ( $P=0.023, P=0.009, P=0.002$ respectively), as did the overall 'MUST' score $(P=0 \cdot 009)$.

Discharge destination and readmissions. Although a greater proportion of high-risk patients $(51 \%)$ did not return

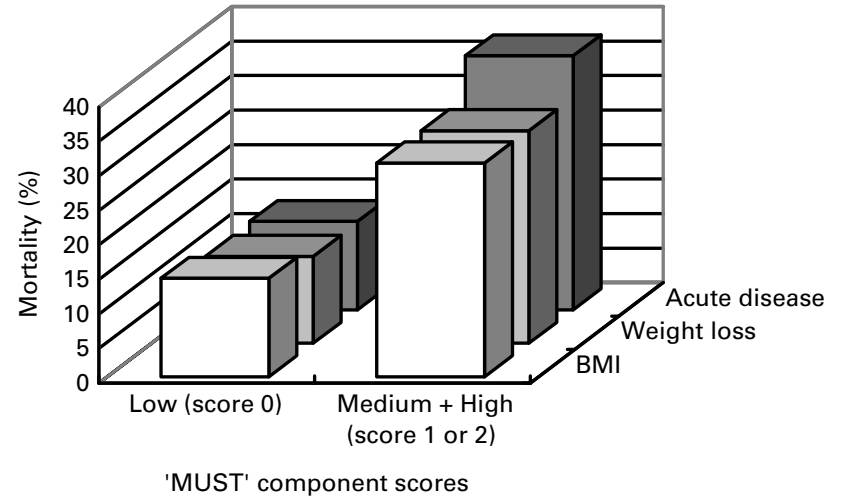

Fig. 3. In-hospital mortality according to each 'Malnutrition Universal Screening Tool' ('MUST') component (BMI, $P=0.02$; percentage weight loss, $P=0.007$; acute disease, $P=0.001$ ). For details of procedures, see p. 326 .

home after hospital discharge (v. $40 \%$ of the low-risk group), this was not significant. There was also no difference in the proportion of patients readmitted to hospital $(29 \% \mathrm{v}$. $23 \%$ ), the number of readmissions to hospital (median 0 (range 0-2) for all risk categories) or the number of days spent in hospital on readmissions $(0$ (range $0-48) \mathrm{d} v$. 0 (range 0-52) d) up to 3 months post-discharge, according to 'MUST' category (low $v$. medium + high).

Malnutrition risk and clinical outcome of patients who could v. could not be weighed

Patients who could not have their weight measured during screening $(n 66)$ had a greater prevalence of malnutrition $(70 \%)$ than those who could be weighed (49\%) $\left(P=0 \cdot 01, \chi^{2}\right.$ 6.6). Malnutrition prevalence (medium + high risk) was greatest $(89 \%)$ in the thirty-five patients for whom 'MUST' subjective criteria were used to calculate risk (patients who could not be weighed with no recalled weight). These patients were more likely to be malnourished and to have significantly poorer outcome (higher mortality, longer length of stay, greater need for support post-discharge) than those who could be weighed (Table 2).

Predictive validity of 'MUST' in patients who could v. could not be weighed

The relationship of 'MUST' malnutrition risk (low $v$. medium + high) to increased mortality was observed both in patients whose weight could and could not be measured during screening (Fig. 4). In patients who could not be weighed and whose malnutrition risk was categorised using recalled weight or subjective criteria ( $n$ 66), those at malnutrition risk (medium + high 'MUST') had significantly greater mortality compared with those who were at low risk $(34.8 \% \mathrm{v}$. $5.3 \% ; P=0.014, \chi^{2}$ 6.07; Fig. 3) (for patients screened using only subjective criteria, the mortality in medium + high- $v$. low-risk patients was $45.2 \% v$. $0 \%$, respectively $(n 35)$; $P=0.08, \chi^{2} 3.01$; data not shown in Fig. 3).

There were no significant differences in mortality between low-risk patients who could and could not be weighed during screening with 'MUST' (Fig. 4). However, there was a tendency (not significant) for medium + high-risk patients who could not be weighed to have higher mortality than those who could be weighed (Fig. 4). 
Table 2. Malnutrition risk and clinical outcome of patients with no measured or recalled weight*

\begin{tabular}{lccc}
\hline & No weight $(n$ 35) & Weight $(n$ 115) & $P$ value \\
\hline Percentage at risk of malnutrition & 88.6 & 48.7 & $0.0005 \dagger$ \\
Medium + high 'MUST' risk & $14.3+74.3$ & $17.4+31.3$ & - \\
Percentage in-hospital mortality & 40 & 12.2 & $0.002 \dagger$ \\
Median length of stay in days & 50 & 17 & $0.0005 \ddagger$ \\
$95 \% \mathrm{Cl}$ & 13,87 & 37,20 & - \\
Percentage discharged to & 90 & & $0.0005 \S$ \\
$\quad$ destination other than home & & &
\end{tabular}

'MUST', 'Malnutrition Universal Screening Tool'.

* Patients with no measured or recalled weight older ( $87 v$ v. 85 years, $P=0.044)$. For details of procedures, see p. 326.

$\dagger$ Binary logistic regression (adjusted for age and sex, $n$ 150).

‡ Kaplan-Meier survival analysis (log rank test, twenty-eight of 150 cases censored).

$\S$ Binary logistic regression (adjusted for age and sex, $n$ 122; 101 and 21 per group).

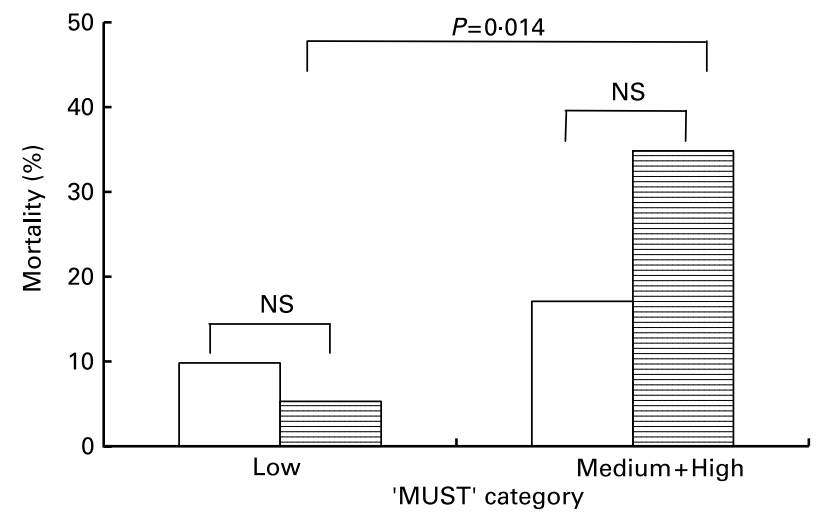

Fig. 4. Mortality according to 'Malnutrition Universal Screening Tool' ('MUST') for patients who could ( $\square$ ) and could not (目) have weight measured. There were no significant differences in age between groups. For details of procedures, see p. 326.

\section{Discussion}

This is the first study to indicate the practicality and predictive validity of 'MUST' in a group of acutely ill, hospitalised elderly patients. The ability of 'MUST' to screen all of the elderly patients in the present study provides more complete information than many other studies (Kelly et al. 2000; Powell-Tuck \& Hennessy, 2003) of the prevalence of malnutrition (58\% at risk) and its relationship to poor clinical outcome in all patients (Figs 2 and 3), including those who can and cannot be weighed as part of screening (Fig. 4).

The finding that 'MUST' was easy to use and took 5 min or less to complete in this elderly patient group was in line with previous observations about the practicality of 'MUST' in other types of patients (Stratton et al. 2004). The present study also confirmed previous observations using other screening tools that malnutrition is a substantial problem in this patient group (58\%) (Stratton et al. 2003b). However, one issue, which has often been overlooked in previous screening trials, especially in elderly care wards, is that a significant proportion of patients cannot be screened as they cannot be weighed or have their height measured (e.g. many tools require BMI to be completed during screening). Consequently, studies of malnutrition prevalence and its relationship to outcome have often excluded patients who could not be weighed (Kelly et al. 2000; Powell-Tuck \& Hennessy, 2003). Crucially, however, in the pre- sent study, all consecutive patients could be screened with 'MUST' ( $n$ 150) and malnutrition risk documented, enabling a more complete picture of the prevalence of malnutrition and its impact on clinical outcome to be assessed for all patients in this group. This was despite there being $44 \%$ ( $n$ 66) of patients who could not be weighed. These individuals ( $n$ 66) could still be screened using 'MUST' due to the use of recalled and subjective criteria incorporated within 'MUST' (for reliability see Elia, 2003; Stratton et al. 2003a). Measurement of height was also difficult in this group (possible in eighteen of the 150 patients $-12 \%$ of the whole group) and so recalled/documented heights and surrogate measures to calculate height enabled BMI to be calculated for those for whom a weight could be obtained (Elia, 2003).

The present study also investigated the predictive validity of 'MUST'. In this patient group, 'MUST' risk predicted some clinical outcomes, namely mortality both in hospital and 3 and 6 months after discharge and length of hospital stay (Fig. 2). Discharge to destinations other than home and readmission rates were not significantly different in those with malnutrition (medium + high risk). However, it is widely acknowledged that admission and discharge to institutions are affected by many factors, often socially determined and which may be independent of nutritional status. Alternatively, in the present study, the sample size may have been inadequate to detect differences. The individual components of 'MUST' were significantly related to outcome (Fig. 3). In addition, the relationship between 'MUST' risk and outcome (e.g. in-hospital mortality) persisted in those patients for whom only recalled or subjective data were used to categorise 'MUST' risk (Fig. 4). Importantly, the present study also highlighted that patients who could not have their weight measured (nor reliable data on recent weight obtained) were significantly more likely to be at malnutrition risk and to have a poorer outcome (Table 2).

The high prevalence of malnutrition $(58 \%)$ and the associated poorer clinical outcome of this elderly patient group is concerning. The present findings support the consensus of recommendations made by many national and international bodies that routine screening to identify malnutrition should be carried out so that patients can be treated (Elia et al. 2005). Indeed, systematic reviews and meta-analyses have suggested that the use of oral nutritional support in many patient groups, including the elderly, can significantly improve clinical outcome (mortality, complication rates) and body function in the treatment of malnutrition 
(Potter et al. 1998; Stratton \& Elia, 1999; Potter, 2001; Stratton et al. 2003b), despite the problems with anorexia and early satiety in the elderly (Morley, 1996).

Although one of the main aims of the present study was to assess the predictive validity of 'MUST', other types of validity for screening tools are also important, as a standard reference method to diagnose malnutrition is lacking. Indeed, in addition to being reliable and reproducible, 'MUST' has concurrent validity with other published tools (Elia, 2003; Stratton et al. 2004). One issue that remains to be fully addressed by well-designed randomised controlled trials is the impact of intervening with a screening programme (that includes screening and treatment), on the care and clinical outcome of patients, including those who are and are not identified as at risk (Elia et al. 2005). Although improvements in outcome have been observed in patients treated for risk of malnutrition (after identification with screening; Johansen et al. 2004), randomised controlled trials that study the effects of screening and associated care plans in all patients (with and without malnutrition) are lacking.

In conclusion, this is the first study to show that 'MUST' has predictive validity in the elderly hospitalised population, with regard to mortality, both in hospital and after discharge, and length of hospital stay. In the present study, the individual components of 'MUST' related to outcome (mortality), and the relationship between 'MUST' and poorer outcome persisted when only recalled or subjective data could be used in 'MUST' risk categorisation. 'MUST' could be used to screen all of the elderly care inpatients, in whom the prevalence of malnutrition was high $(58 \%)$. Those who could only be screened using 'MUST' subjective criteria (e.g. no measured or recalled weight) had the highest prevalence of malnutrition $(89 \%)$ and the poorest clinical outcome. The present findings highlight the importance of using a method to identify malnutrition that can be used in all patients in routine practice so that a suitable, evidence-based treatment plan can be implemented, with a view to improving clinical outcome.

\section{Acknowledgements}

Some of the data in this paper were previously presented as an abstract at the European Society for Clinical Nutrition and Metabolism (ESPEN). This work was supported by Southampton NHS Research and Development.

\section{References}

ASPEN (1995) Standards for nutrition support: hospitalised patients. Nutr Clin Pract 10, 208-219.

British Dietetic Association (1999) Nutrition Screening Tools. Professional Development Committee Briefing Paper no. 9. Birmingham: British Dietetic Association.

Council of Europe (2002) Food and Nutritional Care in Hospitals: How to Prevent Undernutrition. Report and Recommendations of the Committee of Experts on Nutrition, Food Safety and Consumer Protection. Strasbourg: Council of Europe.

Department of Health (2001) Essence of Care - Patient Focussed Benchmarking for Healthcare Practitioners. London: Department of Health.

Detsky AS, McLaughlin JR, Baker JP, Johnston N, Whittaker S, Mendelson RA \& Jeejeebhoy KN (1987) What is Subjective Global Assessment of nutritional status?" J Parent Enteral Nutr 11, 8-13.

Elia M (chairman and editor) (2000) Guidelines for Detection and Management of Malnutrition. Malnutrition Advisory Group,
Standing Committee of British Association for Parenteral and Enteral Nutrition. Maidenhead: BAPEN.

Elia M (2003) Screening for Malnutrition: A Multidisciplinary Responsibility. Development and Use of the Malnutrition Universal Screening Tool ('MUST') for Adults. Redditch: BAPEN.

Elia M, Zellipour L \& Stratton RJ (2005) To screen or not to screen for adult malnutrition?" Clin Nutr 24, 867-884.

Guigoz Y, Vellas B \& Garry PJ (1996) Assessing the nutritional status of the elderly: the Mini Nutritional Assessment as part of the geriatric evaluation. Nutr Rev 54, S59-S65.

Johansen N, Kondrup J, Plum LM, Bak L, Norregaard P, Bunch E, Baernthsen H, Andersen JR, Larsen IH \& Martinsen A (2004) Effect of nutritional support on clinical outcome in patients at nutritional risk. Clin Nutr 23, 539-550.

Kelly IE, Tessier S, Cahill A, Morris SE, Crumley A, McLaughlin D, McKee RF \& Lean MEJ (2000) Still hungry in hospital: identifying malnutrition in acute hospital admissions. Q J Med 93, 93-98.

Kondrup J, Allison SP, Elia M, Vellas B \& Plauth M (2003) ESPEN guidelines for nutrition screening 2002. Clin Nutr 22, 415-421.

Kondrup J, Rasmussen HH, Hamberg O, Stanga Z \& ad hoc ESPEN Working Group (2003) Nutritional Risk Screening (NRS 2002): a new method based on an analysis of controlled clinical trials. Clin Nutr 22, 321-336.

McWhirter JP \& Pennington CR (1994) Incidence and recognition of malnutrition in hospital. Br Med J 308, 945-948.

Morley JE (1996) Anorexia in older persons. Epidemiology and optimal treatment. Drugs Aging 8, 134-155.

Morley JE (1998) Protein-energy malnutrition in older subjects. Proc Nutr Soc 57, 587-592.

Mowe M \& Bohmer T (1991) The prevalence of undiagnosed proteincalorie undernutrition in a population of hospitalised elderly patients. J Am Geriatr Soc 39, 1089-1092.

Murphy MC, Brooks CN, New SA \& Lumbers ML (2000) The use of the Mini-Nutritional Assessment (MNA) tool in elderly orthopaedic patients. Eur J Clin Nutr 54, 555-562.

NHS Quality Improvement Scotland (2003) Clinical Standards: Food, Fluid and Nutritional Care in Hospitals. Edinburgh: NHS Quality Improvement Scotland.

Potter JM (2001) Oral supplements in the elderly. Curr Opin Clin Nutr Metab Care 4, 21-28.

Potter J, Langhorne P \& Roberts M (1998) Routine protein energy supplementation in adults: systematic review. Br Med J 317, 495-501.

Powell-Tuck J \& Hennessy EM (2003) A comparison of mid upper arm circumference, body mass index and weight loss as indices of undernutrition in acutely hospitalized patients. Clin Nutr 22, 307-312.

Reilly HM, Martineau JK, Moran A \& Kennedy H (1995) Nutritional screening - evaluation and implementation of a simple nutrition risk score. Clin Nutr 14, 269-273.

Royal College of Physicians (2002) Nutrition and Patients. A Doctor's Responsibility. Report of a Working Party of the Royal College of Physicians. London: Royal College of Physicians.

Stratton RJ, Dixon R, Longmore D, Stroud M \& Elia M (2003a) Effect of recalled weight and height on malnutrition risk. Clin Nutr 22, S9-S10.

Stratton RJ \& Elia M (1999) A critical, systematic analysis of the use of oral nutritional supplements in the community. Clin Nutr 18, 29-84.

Stratton RJ, Green CJ \& Elia M (2003b) Disease-related Malnutrition: An Evidence Based Approach to Treatment. Wallingford: CABI Publishing.

Stratton RJ, Hackston A, Longmore D, Dixon R, Price S, Stroud M, King C \& Elia M (2004) Malnutrition in hospital outpatients and inpatients: prevalence, concurrent validity and ease of use of the 'Malnutrition Universal Screening Tool' ('MUST') for adults. Br J Nutr 92, 799-808.

Todorovic V, Russell C, Stratton R, Ward J \& Elia M (2003) The 'MUST' Explanatory Booklet. Redditch: BAPEN.

Waitzberg DL, Caiaffa WT \& Correia ITD (2001) Hospital malnutrition: the Brazilian national survey (IBRANUTRI): a study of 4000 patients. Nutrition 17, 573-580. 